Abstract

\title{
Insights into the Activity of Second-Generation Maturation Inhibitors against HIV Clade $C^{+}$
}

\author{
Dibya Ghimire, Yuvraj KC and Ritu Gaur * \\ Faculty of Life Sciences and Biotechnology, South Asian University, New Delhi 110021, India; \\ dibya.ghimire4@gmail.com (D.G.); yuvraj.bhu2009@gmail.com (Y.K.) \\ * Correspondence: rgaur@sau.ac.in \\ + Presented at Viruses 2020-Novel Concepts in Virology, Barcelona, Spain, 5-7 February 2020. \\ Published: 16 June 2020
}

\begin{abstract}
Maturation inhibitors represent a new underdeveloped class of antiretroviral agents that block virus maturation by binding to the target of protease (PR)-Gag precursor (Pr55Gag). Development of a maturation inhibitor is based on a number of small molecules that are capable of blocking the cleavage event between p24-CA and spacer peptide 1. The bulk of the literature on HIV antiretroviral therapy and drug resistance has primarily been derived from HIV-1B, and relatively less is known about the context of HIV-1C that is responsible for more than 95\% of HIV infections of India and half of these infections globally. We and others have shown that the presence of maturation inhibitor resistance mutations would make HIV-1B particles either less fit or dependent on these drugs to replicate. By contrast, it is unclear how HIV-1C would naturally acquire these mutations yet remain replication competent in the absence of the selective pressure of maturation inhibitors. Bevirimat, the first-in-class MI, was found to be inactive against HIV-C due to polymorphisms in the SP1 region. We have identified novel second generation of HIV maturation inhibitors with high potency against $\mathrm{HIV}$ clade $\mathrm{C}$ ( $\mathrm{IC}_{50}$ values in the low $\mathrm{nM}$ range). The mutations identified during selection experiments revealed the putative binding pocket of these compounds on HIV-1 subtype C Gag. While working on the role of CA-C terminal domain (CA-CTD) domain in HIV-1 Gag assembly and release, we have found that core glycine-rich residues of the $\beta$-turn motif are crucial in Gag-membrane binding, multimerization, and assembly. Furthermore, we have identified a novel mutation in CA-CTD that is dependent on both classes of MIs. In conclusion, our studies provide insights into the mechanistic action of MIs on HIV-1 Gag processing and stabilization.
\end{abstract}

Keywords: HIV-1; subtype C; Gag; antiviral; maturation

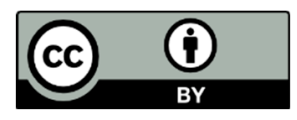

(C) 2020 by the authors. Licensee MDPI, Basel, Switzerland. This article is an open access article distributed under the terms and conditions of the Creative Commons Attribution (CC BY) license (http://creativecommons.org/licenses/by/4.0/). 